\title{
Combination of the neutrophil to lymphocyte ratio and serum toluidine red unheated serum test titer as a predictor of neurosyphilis in HIV-negative patients
}

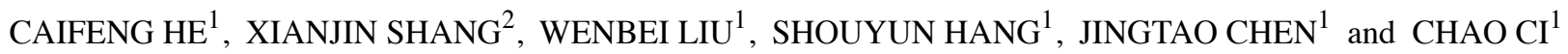 \\ Departments of ${ }^{1}$ Dermatology and ${ }^{2}$ Neurology, Yijishan Hospital of Wannan Medical College, \\ Wuhu, Anhui 241001, P.R. China
}

Received October 17, 2019; Accepted November 20, 2020

DOI: $10.3892 /$ etm.2021.9616

\begin{abstract}
The present study explored the associations of the neutrophil to lymphocyte ratio (NLR) and the serum toluidine red unheated serum test (TRUST) titer with neurosyphilis (NS). The present retrospective study examined $87 \mathrm{NS}$ patients and 80 Non-NS patients from an HIV-negative cohort and 1:1 age- and gender-matched healthy controls. The results demonstrated that the NLR was increased in both NS and Non-NS groups compared with that in the healthy controls $(\mathrm{P}<0.001$ and $\mathrm{P}=0.01$, respectively). The NLR and serum TRUST titer in the NS group were significantly higher than those in the Non-NS group $(\mathrm{P}=0.004$ and $\mathrm{P}<0.001$, respectively). The NLR was positively correlated with the serum TRUST titer $(r=0.298$, $\mathrm{P}<0.001)$. Age, elevated NLR and serum TRUST titer were distinctly associated with NS by binomial logistic regression analysis [odds ratio $(\mathrm{OR})=1.10, \mathrm{P}<0.001 ; \mathrm{OR}=1.36, \mathrm{P}=0.028$; $\mathrm{OR}=3.07, \mathrm{P}<0.001$; respectively]. The cut-off values for the NLR and serum TRUST titer were 1.97 and 1:8, respectively. A significantly higher sensitivity of $90.8 \%$ was obtained for screening out NS with a combination of the NLR and serum TRUST titer compared with each test alone. Age, elevated NLR and serum TRUST titer were associated with NS. The combination of NLR and serum TRUST titer is a potential predictor for NS, and the reduced NLR and serum TRUST titer at the 6-month follow up suggested that the NLR and serum TRUST titer were biomarkers for monitoring the disease course.
\end{abstract}

\section{Introduction}

Neurosyphilis (NS) is a neurological disorder of the central nervous system caused by Treponema pallidum (T. pallidum)

Correspondence to: Dr Chao Ci, Department of Dermatology, Yijishan Hospital of Wannan Medical College, 2 Zhe Shan West Road, Wuhu, Anhui 241001, P.R. China

E-mail: cichao8911@163.com

Key words: neurosyphilis, neutrophil to lymphocyte ratio, toluidine red unheated serum test, predictor infection, which may occur at any stage of syphilis. NS is named 'the great imitator' due to various clinical manifestations such as headache, progressive cognitive decline and stroke; however, patients may also be asymptomatic (1). Due to these varied symptoms, NS may be easily misdiagnosed or ignored. Clinical abnormalities are more likely to resolve in early NS than in late NS; therefore, early recognition of NS is an important issue. Currently, the diagnosis of NS relies on clinical and laboratory results. The cerebrospinal fluid venereal disease research laboratory (CSF-VDRL) tests, the CSF rapid plasma reagin (RPR) or CSF-serum toluidine red unheated serum test (TRUST) are not reactive in all cases of NS $(2,3)$. Lumbar puncture (LP) for CSF examinations is currently the imperfect gold standard for NS. However, differing opinions of clinicians, poor patient acceptance and invasiveness are limitations for using LP as a disease course monitoring tool. Thus, simple and noninvasive indices based on routine laboratory tests to monitor the disease course are of great importance.

The neutrophil to lymphocyte ratio (NLR) is a stable complete blood count parameter that is used as a marker of inflammation for diseases such as stroke, systemic lupus erythematosus, pulmonary tuberculosis, psoriasis arthritis and dermatomyositis (4-8). A positive association of the NLR with disease activity has emerged; the NLR is positively associated with lupus nephritis, psoriatic arthritis and pulmonary involvement in patients with dermatomyositis $(4,6,7)$. Furthermore, associations of the NLR with other conditions, such as Hashimoto's disease, diabetes mellitus, thyroid nodules, cardiac conditions, numerous cancer types and vitamin D deficiency has been reported (9-16); however, the association between the NLR and NS has remained to be determined.

All individuals with syphilis and HIV co-infection are recommended to undergo LP (17); however, whether and when to perform LP in patients with HIV-negative NS is controversial. A recent study reported that a serum T. pallidum agglutination assay (TPPA) titer $\geq 1: 2,560$, a serum RPR titer $\geq 1: 4$ and increased levels of serum creatine kinase are able to predict symptomatic NS (18). However, indicators for symptomatic NS and asymptomatic NS prior to LP in HIV-negative patients have remained to be determined. In the present study, it was assessed whether NLRs and serum TRUST titers are 
different between NS and Non-NS patients. Furthermore, the performance of the two markers for predicting NS was also determined.

\section{Subjects and methods}

Subjects. NS and Non-NS patients, as well as age- and gender-matched healthy controls, who attended Yijishan Hospital of Wannan Medical College (Wuhu, China) between January 2015 and August 2018, were retrospectively reviewed. The NS patients met all of the following requirements: i) Serum TPPA and TRUST titer were positive; ii) serological tests were combined with CSF-TRUST positivity; or CSF white blood cell (WBC) count $>5$ cells $/ \mu 1$ or CSF-protein $>45 \mathrm{mg} / \mathrm{dl}$ and CSF-TPPA reactivity (10). Non-NS was defined as a seropositive for TPPA and TRUST but a negative for the CSF-TRUST and CSF-TPPA with all of the following: i) CSF WBC $\leq 5$ cells $/ \mu l$ and CSF protein $\leq 45 \mathrm{mg} / \mathrm{dl}$; ii) absence of any characteristic symptoms or signs of NS. Healthy controls who underwent normal health check-ups that revealed no diseases with a normal erythrocyte sedimentation rate (ESR) and C-reactive protein (CRP) levels were recruited from the database of Yijishan Hospital of Wannan Medical College between January 2015 and August 2018 (Wuhu, China).

The following exclusion criteria were applied: Pregnancy, incomplete documentation, the existence of other chronic inflammatory disorders (e.g., autoimmunity) and other infectious diseases (e.g., tuberculosis, urinary tract infection and pneumonia), and treatment with anti-inflammatory or immunosuppressive drugs or antibiotics within the last 6 months. All patients were positive for serum TRUST and TPPA and negative for HIV.

Data. Demographic, clinical and laboratory data were retrieved from the hospital's information system. The following data were included: Age, sex, complete blood count, serum TRUST, CSF-WBC count, CSF protein level, CSF-TPPA, CSF-TRUST and serological testing (TRUST, TPPA). The above-mentioned data of 24 NS patients were collected after treatment at a 6-month follow-up. The NLR was calculated as the neutrophil count divided by the lymphocyte count.

Statistical analysis. The TRUST titers were transferred to $\log _{2}$ transformations ( $\log _{2} 1 /$ TRUST titer) for analysis. Quantitative variables are presented as the mean \pm standard deviation or median (interquartile range) as appropriate. Qualitative variables are expressed as the number and percentage. Univariate analyses were performed by Student's t-test or the Mann-Whitney U-test for quantitative variables as appropriate. Qualitative variables were compared by the $\chi^{2}$ test or Fisher's exact test as appropriate. Independent predictors for NS were analyzed using standard binomial logistic regression analysis. The Spearman test was performed to estimate the correlation between variables. Receiver operating characteristic (ROC) curves were used to identify cutoff values of the NLR and the serum TRUST titer for discriminating between NS and Non-NS. To evaluate the changes in parameters between the baseline and the designated follow-up time-point, a two-tailed Wilcoxon rank-sum test was employed. Statistical analysis was performed using SPSS Statistics 22.0 (IBM Corp). P-values were two-sided and $\mathrm{P}<0.05$ was considered to indicate statistical significance.

\section{Results}

Comparison of variables between NS patients or Non-NS patients and healthy controls. The demographic data and laboratory findings of 87 NS patients, 80 Non-NS patients and 1:1 age- and gender-matched healthy individuals are presented in Table SI. The mean age of NS group was $51.03 \pm 13.09$ years (range, 21-81), and the group included 54 males and 33 females. The mean age of Non-NS patients was $35.20 \pm 11.26$ years (range, 20-76), and these patients included 18 males and 62 females. The mean age in the healthy control patient group that was compared with the NS group was $49.53 \pm 11.18$ years (range, 21-68), the sex distribution was 48 men and 39 women. The mean age of the healthy control patients that were compared to the Non-NS group was $34.65 \pm 10.30$ years (range, 20-67), the sex distribution was 19 men and 61 women. Peri-WBC, neutrophil, ESR and the NLR were significantly higher in the NS group compared with the healthy controls $(\mathrm{P}<0.001$; except for the peri-WBC count, $\mathrm{P}=0.01)$. Lymphocyte counts were lower in the NS group than in the healthy controls $(\mathrm{P}<0.001)$. The peri-WBC and neutrophil counts were higher in the Non-NS group than that in healthy controls $(\mathrm{P}<0.001)$. However, the lymphocyte counts were not significantly different between the Non-NS group and healthy controls $(\mathrm{P}=0.741)$. The NLR was higher in the Non-NS groups than that in the healthy group $(\mathrm{P}=0.01)$. No significant difference in CRP was observed between the NS or the Non-NS and healthy controls $(\mathrm{P}>0.05)$.

Comparison of laboratory parameters between NS and Non-NS patients. In comparison to the Non-NS group, in NS patients male sex was predominant, the mean age was older and ESR was higher in the NS group $(\mathrm{P}<0.001)$, while no significant difference was observed for CRP $(P>0.05)$. The median serum TRUST titer was significantly higher in the NS group compared with that in the Non-NS group [1:16 (1:8-1:32) vs. 1:2 (1:2-1:4), $\mathrm{P}<0.001)]$. Lymphocyte counts in NS patients were significantly decreased compared with those in Non-NS patients $(1.58 \pm 0.71$ vs. $1.98 \pm 0.72, \mathrm{P}<0.001)$. Furthermore, the NLR in the NS group was significantly higher compared with that in the Non-NS group ( $\mathrm{P}=0.004$; Table I).

Predictive value of the NLR and serum TRUST titer. Binomial logistic regression analysis indicated a significant association of age, NLR and serum TRUST titer with NS [odds ratio $(\mathrm{OR})=1.099,95 \% \mathrm{CI}=1.052-1.149, \mathrm{P}<0.001$; OR $=1.363$, 95\% $\mathrm{CI}=1.035-1.795, \mathrm{P}=0.028$; and $\mathrm{OR}=3.065,95 \% \mathrm{CI}=2.094-4.487$, $\mathrm{P}<0.001$, respectively; Table II]. The ESR was not included in a further logistic regression analysis due to incomplete data. Spearman's rank correlation analysis indicated that the NLR in patients with NS was positively correlated with the serum TRUST titer ( $\mathrm{r}=0.288, \mathrm{P}<0.001$; Fig. 1). ROC curve analyses indicated that the area under the curve values of the NLR and serum TRUST titer were $0.628(95 \% \mathrm{CI}=0.542-0.714 ; \mathrm{P}=0.01)$ and 0.882 (95\% CI=0.798-0.911; $\mathrm{P}<0.001$ ), respectively (Fig. 2); the optimal cut-off values of the NLR and serum TRUST titer to predict NS were 1.97 (79.3\% sensitivity, $52.5 \%$ specificity) 
Table I. Comparison of patients with NS and non-NS.

\begin{tabular}{lccc}
\hline Parameter & NS $(\mathrm{n}=87)$ & Non-NS $(\mathrm{n}=80)$ & P-value \\
\hline Age (years) & $51.03 \pm 13.09$ & $35.20 \pm 11.26$ & $<0.001$ \\
Male sex & $54(62.1)$ & $18(22.5)$ & $<0.001$ \\
Serum-TRUST & $1: 16(1: 8-1: 32)$ & $1: 2(1: 2-1: 4)$ & $<0.001$ \\
CSF-WBC & $12.89 \pm 14.95$ & $5.26 \pm 1.75$ & $<0.001$ \\
CSF-protein (mg/dl) & $62.99 \pm 43.32$ & $30.45 \pm 11.18$ & $<0.001$ \\
CSF-TRUST & $1: 1($ negative-1:4) & Negative & $<0.001$ \\
Peri-WBC $\left(x 10^{9} / 1\right)$ & $6.93 \pm 2.62$ & $6.83 \pm 1.77$ & 0.61 \\
Neutrophil & $4.59 \pm 2.61$ & $4.27 \pm 1.53$ & 0.72 \\
Lymphocyte & $1.58 \pm 0.71$ & $1.98 \pm 0.72$ & $<0.001$ \\
NLR $_{\text {ESR }}$ & $2.87(2.04-3.89)$ & $1.89(1.50-3.37)$ & 0.004 \\
CRP $^{\mathrm{b}}$ & $16.28 \pm 16.98$ & $6.07 \pm 4.24$ & $3.38 \pm 1.34$ \\
\hline
\end{tabular}

Values are expressed as the mean \pm standard deviation, $\mathrm{n}(\%)$ or the median (interquartile range). ${ }^{\mathrm{a} D e t e r m i n e d ~ i n ~} 67$ of 87 patients with NS and 52 of 80 patients with non-NS. 'betermined in 42 of 87 patients with NS and 36 of 80 patients with non-NS. NLR, neutrophil-to-lymphocyte ratio; serum-TRUST, serum toluidine red unheated serum test; CSF, cerebrospinal fluid; WBC, white blood cell; ESR, erythrocyte sedimentation rate; CRP, C-reactive protein; NS, neurosyphilis.

Table II Binomial logistic regression model for the presence of neurosyphilis.

\begin{tabular}{lccr}
\hline Variable & OR & $95 \%$ CI & P-value \\
\hline Age & 1.099 & $1.052-1.149$ & $<0.001$ \\
NLR & 1.363 & $1.035-1.795$ & 0.028 \\
Serum TRUST titer & 3.065 & $2.094-4.487$ & $<0.001$ \\
Lymphocyte & 2.024 & $0.869-4.715$ & 0.102 \\
\hline
\end{tabular}

OR, odds ratio; CI, Confidence interval; NLR, neutrophil-tolymphocyte ratio; TRUST, toluidine red unheated serum test.

and 1:8 (79.3\% sensitivity, 87.5\% specificity), respectively. For the NS group, $67.8 \%$ of patients had a high NLR and serum TRUST titer and $9.2 \%$ had a low level, while $23.0 \%$ of patients had discordant levels. The serum TRUST test with the NLR test was also combined in a parallel and serial testing format. In the parallel testing format (with either serum TRUST titer $\geq 1: 8$ or NLR $\geq 1.97$, or both), $90.8 \%$ sensitivity and $50.0 \%$ specificity were obtained for predicting NS. In the serial testing format (with serum TRUST titer $\geq 1: 8$ and NLR $\geq 1.97$ ), $67.8 \%$ sensitivity and $90.0 \%$ specificity were obtained for predicting NS. In addition, the NLR, serum TRUST titer, CSF-WBC count, CSF-protein level and CSF-TRUST titer parameters were significantly decreased at the 6-month follow-up after treatment with penicillin $(\mathrm{P}<0.05$; Table SII).

\section{Discussion}

The present study indicated that age, the NLR and serum TRUST titer were independent risk factors of NS, i.e., elevated NLR and serum TRUST titer were able to predict the presence of NS. Furthermore, the NLR was positively associated with

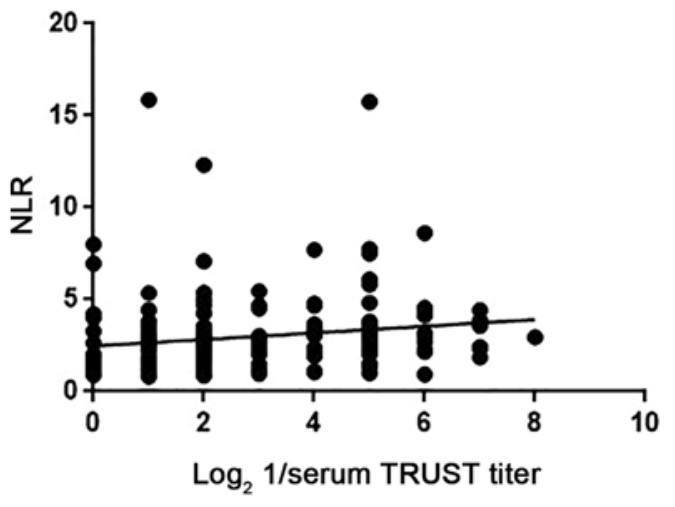

Figure 1. Correlations of $\log _{2} 1 /$ serum TRUST titer with NLR in NS patients. TRUST, toluidine red unheated serum test titer; NLR, neutrophil-to-lymphocyte ratio; NS, neurosyphilis.

the serum TRUST titer and was reduced when the clinical manifestations were resolved and the serum TRUST titer and CSF parameters were reduced. The cut-off values for the serum TRUST titer and the NLR were 1:8 and 1.97, respectively. Combination of the NLR and serum TRUST titer had a substantially higher sensitivity (90.8\%) for identifying NS than the NLR or serum TRUST titer alone.

The NLR, as a marker of chronic systemic inflammation, is a prognostic indicator for numerous cancer types, acute stroke, multiple sclerosis and Parkinson's disease $(5,9,12,19,20)$, while the NLR is used for the diagnosis of numerous inflammatory diseases, including subacute thyroiditis, psoriatic arthritis and lupus nephritis $(4,7,21)$. Inflammation has a significant role in the pathogenesis of NS. Certain inflammatory factors, including B lymphocyte chemoattractant chemokine (C-X-C motif) ligand 13 and the macrophage migration inhibitory factor, interleukin 10, increased markedly in both the serum and CSF of patients with NS (22-24). In vivo, T. pallidum 

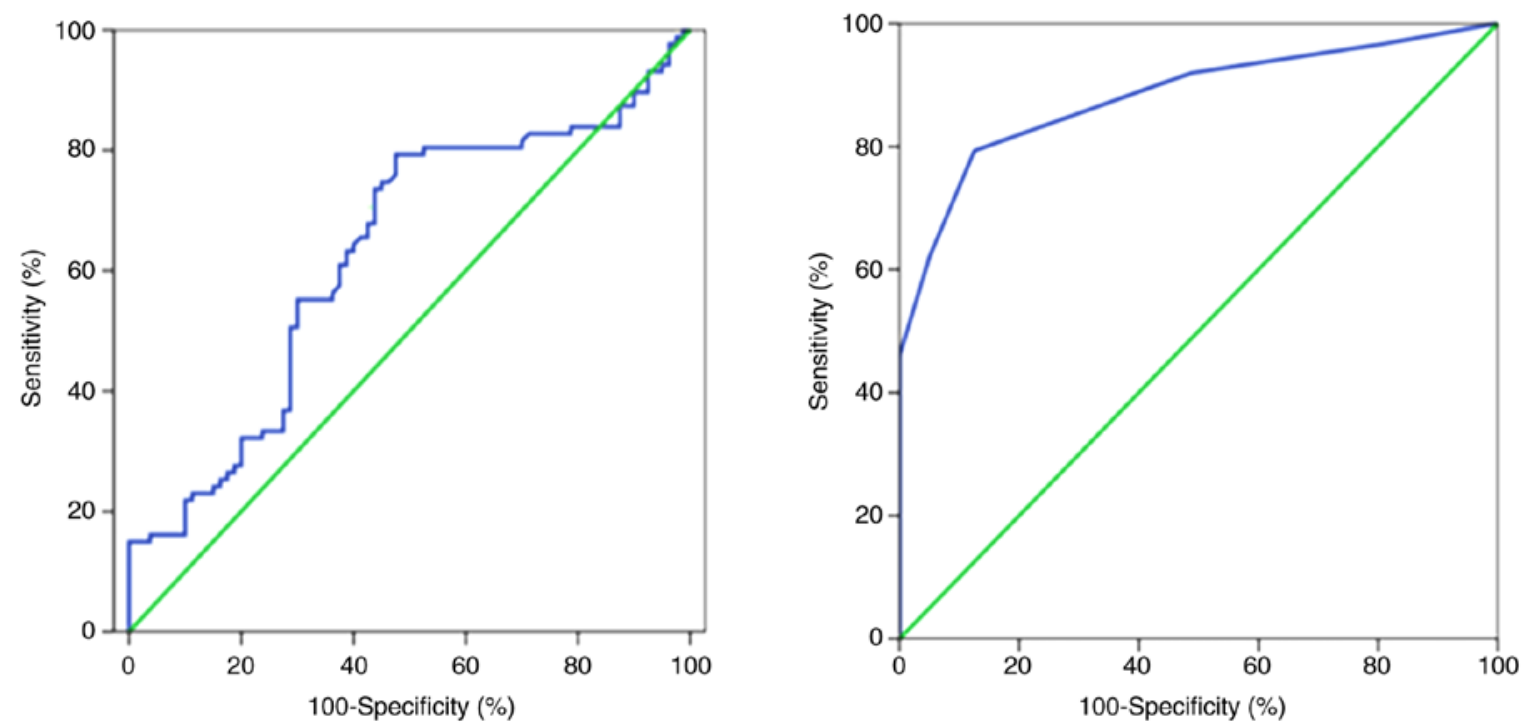

Figure 2. Receiver operating characteristic curves of the neutrophil-to-lymphocyte ratio (left-hand panel) and serum toluidine red unheated serum test titer (right-hand panel) to distinguish between NS and Non-NS. NS, neurosyphilis.

stimulated the expression of inflammatory cytokines in THP-1 cells and induced the transformation of macrophages to a pro-inflammatory M1 macrophage type (25); furthermore, the T. pallidum proteins, including Tp92, Tp0751 and T. pallidum flagellins, may also enhance the release of inflammatory cytokines in HMEC-1/THP-1 cells (26-28). In light of these results, inflammation in the course of NS was confirmed. The ESR in patients with NS was higher than that in patients with Non-NS and it was speculated that differences in the gender distribution and age between NS and Non-NS groups may cause ESR-related differences, as the ESR itself is easily affected by gender and age; however, patients with NS had low and normal levels of CRP, which confirmed that NS is a low-grade inflammatory disease. This, in turn, may account for low lymphocyte counts observed in patients with NS. Furthermore, decreased lymphocyte counts in patients with NS compared with Non-NS patients may cause the NLR to be elevated in patients with NS in the present study, and it was speculated that lymphocyte or lymphocytes-associated signaling pathways may be important for controlling inflammation involved in NS, as T lymphocytes, particularly CD3+CD8+ lymphocytes, natural killer cells and B lymphocytes, are involved in NS $(29,30)$. However, further studies are required to explore the associated molecular mechanisms. In addition, the NLR was determined to be an indicator of disease activity in autoimmune diseases, such as primary Sjögren's syndrome, systemic lupus erythematosus and rheumatoid arthritis $(4,31,32)$. In the present study, binary logistic analysis determined that the NLR was positively associated with the presence of NS; the best NLR cut-off value was 1.97 with $79.3 \%$ sensitivity and $52.5 \%$ specificity and the AUC value was 0.628 . Of note, the NLR was positively associated with the serum TRUST titer, which was determined to be a risk factor of NS, and was decreased at the 6-month follow-up after treatment with penicillin when clinical symptoms and laboratory parameters (serum TRUST titer, CSF-WBC count and CSF-protein level) were resolved. Thus, it was confirmed that the NLR may be an additional marker for diagnosing NS and assessing disease activity.
Risk factors for NS include an age of $\geq 45$ years, high serum RPR titer and HIV $(33,34)$. HIV-positive subjects with RPR titers $\geq 1: 32$ or a CD4+ cell count $\geq 350$ cells $/ \mu 1$ are recommended to undergo LP for CSF examination (35). Conventionally, patients exhibiting signs of at least one of the following, namely neurological, ocular or auricular symptoms, serological failure or serological fast, are recommended to undergo LP to exclude NS (2). In addition, the TRUST test has become commercially and widely available in China. Jiang et al (36) indicated that among HIV-negative patients, those with serum-TRUST titers $\geq 1: 16$ have a higher probability of developing NS. The present study confirmed that age and the serum TRUST titer were risk factors for NS, which is in accordance with previous studies (37). A serum TRUST titer $\geq 1: 8$ with $79.3 \%$ sensitivity and $87.5 \%$ specificity was a strong predictor of NS. Of note, $67.82 \%$ of patients with NS had a concomitantly high serum TRUST titer and a high NLR, indicating that patients with a serum TRUST titer $\geq 1: 8$ and an NLR $\geq 1.97$ are more likely to have NS. Furthermore, a parallel testing and serial testing format were applied, revealing that the NLR plus serum TRUST titer may be a potential predictor of NS (90.8\% sensitivity and $50.0 \%$ specificity, $67.8 \%$ sensitivity and $90.0 \%$ specificity, respectively). Although the sensitivity of a combination of the NLR $(\geq 1.97)$ and serum TRUST titer $(\geq 1: 8)$ was $90.8 \%$ with low specificity $(50 \%)$, there is currently no non-invasive test with this high sensitivity and specificity to predict NS; thus, this may still encourage physicians to perform LP to screen out underlying NS. Furthermore, the sensitivity and specificity obtained with the serial testing format was similar to the CSF-VDRL, which was considered as a standard test with $33.3-70 \%$ sensitivity and $99 \%$ specificity for diagnosing NS $(2,38)$. Thus, this indicated that the NLR $(\geq 1.97)$ and serum TRUST titer $(\geq 1: 8)$ are useful biomarkers for NS.

Non-invasive markers for predicting NS are currently insufficient. In the present study, two easily accessible indices related to NS were assessed and their diagnostic value for NS was evaluated. Several limitations must be acknowledged in the current study. First, the results are applicable to subjects 
with suspected NS. Furthermore, the patients with Non-NS were not stratified according to the stage of syphilis and whether there is a difference between Non-NS at different stages and NS requires to be confirmed with further large samples. Finally, the present study was a single-center retrospective analysis of prospectively collected data, and thus, a prospective, multi-center, large-sample study is warranted.

In conclusion, the combination of the NLR and serum TRUST titer is a useful indicator of NS. The NLR and serum TRUST titer were reduced at the 6 month follow-up with resolved clinical manifestations and CSF laboratory parameters, suggesting that these simple, rapid and easily obtainable prognostic biomarkers may be utilized to monitor the disease course.

\section{Acknowledgements}

Not applicable.

\section{Funding}

This work was supported by the scientific research fund of Wannan Medical College in Anhui Province, Wuhu, China (grant no. WK2017F08 to CH).

\section{Availability of data and materials}

The datasets used and/or analyzed during the current study are available from the corresponding author on reasonable request.

\section{Authors' contributions}

$\mathrm{CH}$ planned and conceived the study. $\mathrm{CH}, \mathrm{XS}$ and $\mathrm{CC}$ collected the data. WL, SH and JC interpreted the data. $\mathrm{CH}$ and $\mathrm{CC}$ wrote and critically revised the manuscript. All authors read and approved the final manuscript.

\section{Ethics approval and consent to participate}

This study was approved by the ethics committee of Yijishan Hospital of Wannan Medical College (Wuhu, China) and all participants provided written informed consent.

\section{Patient consent for publication}

Not applicable.

\section{Competing interests}

The authors declare that they have no competing interests.

\section{References}

1. Zheng D, Zhou D, Zhao Z, Liu Z, Xiao S, Xing Y, Suo WZ and Liu J: The clinical presentation and imaging manifestation of psychosis and dementia in general paresis: A retrospective study of 116 cases. J Neuropsychiatry Clin Neurosci 23: 300-307, 2011.

2. Janier M, Hegyi V, Dupin N, Unemo M, Tiplica GS, Potocnik M, French P and Patel R: 2014 European guideline on the management of syphilis. J Eur Acad Dermatol Venereol 28: 1581-1593, 2014.
3. Marra CM, Tantalo LC, Maxwell CL, Ho EL, Sahi SK and Jones T: The rapid plasma reagin test cannot replace the venereal disease research laboratory test for neurosyphilis diagnosis. Sex Transm Dis 39: 453-457, 2012.

4. Soliman WM, Sherif NM, Ghanima IM and El-Badawy MA: Neutrophil to lymphocyte and platelet to lymphocyte ratios in systemic lupus ery thematosus: Relation with disease activity and lupus nephritis. Reumatol Clin 16: 255-261, 2020.

5. Goyal N, Tsivgoulis G, Chang JJ, Malhotra K, Pandhi A, Ishfaq MF, Alsbrook D, Arthur AS, Elijovich L and Alexandrov AV: Admission neutrophil-to-lymphocyte ratio as a prognostic biomarker of outcomes in large vessel occlusion strokes. Stroke 49: 1985-1987, 2018.

6. Yang W, Wang X, Zhang W, Ying H, Xu Y, Zhang J, Min Q and Chen J: Neutrophil-lymphocyte ratio and platelet-lymphocyte ratio are 2 new inflammatory markers associated with pulmonary involvement and disease activity in patients with dermatomyositis. Clin Chim Acta 465: 11-16, 2017.

7. Kim DS, Shin D, Lee MS, Kim HJ, Kim DY, Kim SM and Lee MG: Assessments of neutrophil to lymphocyte ratio and platelet to lymphocyte ratio in Korean patients with psoriasis vulgaris and psoriatic arthritis. J Dermatol 43: 305-310, 2016

8. Abakay O, Abakay A, Sen HS and Tanrikulu AC: The relationship between inflammatory marker levels and pulmonary tuberculosis severity. Inflammation 38: 691-696, 2015.

9. Russo A, Russano M, Franchina T, Migliorino MR, Aprile G, Mansueto G, Berruti A, Falcone A, Aieta M, Gelibter A, et al: Neutrophil-to-lymphocyte ratio (NLR), platelet-to-lymphocyte ratio (PLR), and outcomes with nivolumab in pretreated non-small cell lung cancer (NSCLC): A large retrospective multicenter study. Adv Ther 37: 1145-1155, 2020.

10. Jiang Y, Weng R, Zhang Y, Fan R, Liu Y, Chen Z, Peng F, Chen Y and Chen X: The performance of rapid plasma reagin (RPR) titer in HIV-negative general paresis after neurosyphilis therapy. BMC Infect Dis 18: 144, 2018.

11. Erkus E, Aktas G, Atak BM, Kocak MZ, Duman TT and Savli H: Haemogram parameters in vitamin D deficiency. J Coll Physicians Surg Pak 28: 779-782, 2018.

12. Sakamoto T, Saito H, Uchinaka EI, Morimoto M, Amisaki M, Tokuyasu N, Honjo S, Ashida K and Fujiwara Y: The combination of neutrophil-to-lymphocyte ratio and serum carbohydrate antigen 19-9 level as a prognostic indicator in patients with recurrent pancreatic cancer. Anticancer Res 38: 5497-5503, 2018.

13. Aktas G, Sit M, Dikbas O, Erkol H, Altinordu R, Erkus E and Savli H: Elevated neutrophil-to-lymphocyte ratio in the diagnosis of Hashimoto's thyroiditis. Rev Assoc Med Bras (1992) 63: 1065-1068, 2017.

14. Ackland GL, Abbott TEF, Cain D, Edwards MR, Sultan P, Karmali SN, Fowler AJ, Whittle JR, MacDonald NJ, Reyes A, et al: Preoperative systemic inflammation and perioperative myocardial injury: Prospective observational multicentre cohort study of patients undergoing non-cardiac surgery. Br J Anaesth 122: 180-187, 2019.

15. Duman TT, Aktas G, Atak BM, Kocak MZ, Erkus E and Savli H: Neutrophil to lymphocyte ratio as an indicative of diabetic control level in type 2 diabetes mellitus. Afr Health Sci 19: 1602-1606, 2019.

16. Sit M, Aktas G, Erkol H, Yaman S, Keyif F and Savli H: Neutrophil to lymphocyte ratio is useful in differentiation of malign and benign thyroid nodules. P R Health Sci J 38: 60-63, 2019.

17. Wong T, Fonseca K, Chernesky MA, Garceau R, Levett PN and Serhir B: Canadian public health laboratory network laboratory guidelines for the diagnosis of neurosyphilis in Canada. Can J Infect Dis Med Microbiol 26 (Suppl A): 18A-22A, 2015.

18. Xiao Y, Tong ML, Liu LL, Lin LR, Chen MJ, Zhang HL, Zheng WH, Li SL, Lin HL, Lin ZF, et al: Novel predictors of neurosyphilis among HIV-negative syphilis patients with neurological symptoms: An observational study. BMC Infect Dis 17: $310,2017$.

19. Hemond CC, Glanz BI, Bakshi R, Chitnis T and Healy BC: The neutrophil-to-lymphocyte and monocyte-to-lymphocyte ratios are independently associated with neurological disability and brain atrophy in multiple sclerosis. BMC Neurol 19: 23. 2019.

20. Haghshomar M, Rahmani F, Hadi Aarabi M, Shahjouei S, Sobhani S and Rahmani M: White matter changes correlates of peripheral neuroinflammation in patients with Parkinson's disease. Neuroscience 403: 70-78, 2017. 
21. Calapkulu M, Sencar ME, Sakiz D, Duger H, Ozturk Unsal I, Ozbek M and Cakal E: The prognostic and diagnostic use of hematological parameters in subacute thyroiditis patients. Endocrine 68: 138-143, 2020.

22. Lin LR, Lin DH, Tong ML, Liu LL, Fan JY, Zhu XZ, Gao K, Chen MJ, Zheng WH, Zhang HL, et al: Macrophage migration inhibitory factor as a novel cerebrospinal fluid marker for neurosyphilis among HIV-negative patients. Clin Chim Acta 463: 103-108, 2016.

23. Hu R, Lu C, Lu S, Hu Y, Ma H, Lai W, Zhu G, Feng P, Lu R and Li Y: Value of CXCL13 in diagnosing asymptomatic neurosyphilis in HIV-infected patients. Int J STD AIDS 27: 141-146, 2016.

24. Pastuszczak M, Jakiela B, Jaworek AK, Wypasek E, Zeman J and Wojas-Pelc A: Association of Interleukin-10 promoter polymorphisms with neurosyphilis. Hum Immunol 76: 469-472, 2015.

25. Lin LR, Gao ZX, Lin Y, Zhu XZ, Liu W, Liu D, Gao K, Tong ML, Zhang HL, Liu LL, et al: Akt, mTOR and NF-KB pathway activation in Treponema pallidum stimulates M1 macrophages. Int Immunopharmacol 59: 181-186, 2018.

26. Xu M, Xie Y, Jiang C, Xiao Y, Kuang X, Wen Y, Tan Y, Tan M, Zhao F, Zeng $\mathrm{T}$ and Wu Y: Treponema pallidum flagellins elicit proinflammatory cytokines from human monocytes via TLR5 signaling pathway. Immunobiology 222: 709-718, 2017.

27. Luo X, Zhang X, Zhao T, Zeng T, Liu W, Deng M and Zhao F: A preliminary study on the proinflammatory mechanisms of Treponema pallidum outer membrane protein Tp92 in human macrophages and HMEC-1 cells. Microb Pathog 110: 176-183, 2017.

28. Liu S, Wang S, Wu Y, Zhao F, Zeng T, Zhang Y, Zhang Q and Gao D: Production of proinflammatory cytokines in the human THP-1 monocyte cell line following induction by Tp0751, a recombinant protein of Treponema pallidum. Sci China Life Sci 53: 229-233, 2010.

29. Liu LL, Chao PL, Zhang HL, Tong ML, Liu GL, Lin LR, Su YH, Wu JY, Dong J, Zheng WH and Yang TC: Analysis of lymphocyte subsets in HIV-negative neurosyphilis patients. Diagn Microbiol Infect Dis 75: 165-168, 2013

30. Yu Q, Cheng Y, Wang Y, Wang C, Lu H, Guan Z, Huang J, Gong W, Shi M, Ni L, et al: Aberrant humoral immune responses in neurosyphilis: CXCL13/CXCR5 play a pivotal role for B-cell recruitment to the cerebrospinal fluid. J Infect Dis 216: 534-544, 2017.
31. Sargin G, Senturk T, Yavasoglu I and Kose R: Relationship between neutrophil-lymphocyte, platelet-lymphocyte ratio and disease activity in rheumatoid arthritis treated with rituximab. Int J Rheum Dis 21: 2122-2127, 2018.

32. Hu ZD, Sun Y, Guo J, Huang YL, Qin BD, Gao Q, Qin Q, Deng AM and Zhong RQ: Red blood cell distribution width and neutrophil/lymphocyte ratio are positively correlated with disease activity in primary Sjogren's syndrome. Clin Biochem 47: 287-290, 2014.

33. Shi M, Peng RR, Gao Z, Zhang S, Lu H, Guan Z, Gao Y, Wang C and Zhou P: Risk profiles of neurosyphilis in HIV-negative patients with primary, secondary and latent syphilis: Implications for clinical intervention. J Eur Acad Dermatol Venereol 30: 659-666, 2016.

34. Pastuszczak M, Zeman J, Jaworek AK and Wojas-Pelc A: Cerebrospinal fluid abnormalities in HIV-negative patients with secondary and early latent syphilis and serum VDRL $\geq 1: 32$. Indian J Dermatol 58: 325, 2013.

35. Marra CM, Maxwell CL, Smith SL, Lukehart SA, Rompalo AM, Eaton M, Stoner BP, Augenbraun M, Barker DE, Corbett JJ, et al: Cerebrospinal fluid abnormalities in patients with syphilis: Association with clinical and laboratory features. J Infect Dis 189: 369-376, 2004

36. Jiang Y, Chen X, Ma X, Yang Y, Peng F and Hu X: The usefulness of toluidine red unheated serum test in the diagnosis of HIV-negative neurosyphilis. Sex Transm Dis 38: 244-245, 2011.

37. Sun JJ, Wang ZY, Shen JY, Shen YZ, Liu L, Wang JR, Zhang RF and Lu HZ: Serum TRSUT Titer $\geq 1$ : 16 is a predictor for neurosyphilis among HIV-infected patients with concurrent syphilis and no neurological symptoms. Medicine (Baltimore) 94: e2023, 2015.

38. Castro R, Prieto ES and da Luz Martins Pereira F: Nontreponemal tests in the diagnosis of neurosyphilis: An evaluation of the Venereal disease research laboratory (VDRL) and the rapid plasma reagin (RPR) tests. J Clin Lab Anal 22: 257-261, 2008.

This work is licensed under a Creative Commons Attribution-NonCommercial-NoDerivatives 4.0 International (CC BY-NC-ND 4.0) License. 